\title{
Glicerina como substituto do milho na nutrição de bovinos e influência na composição de ácidos graxos da carne: revisão
}

\author{
$\underline{\text { Mariana Garcia Ornaghi }}^{1}$; Carlos Emanuel Eiras ${ }^{1}$; Ana Guerrero Barrado ${ }^{2}$; $\underline{\text { Juliana Akamine }}$ \\ Torrecilhas $^{1}$; Rodrigo Augusto Cortêz Passetti ${ }^{1}$; $\underline{\text { Camila Mottin }}^{3}$; $\underline{\text { Ivanor Nunes do Prado }}^{4}$ \\ ${ }^{1}$ Discente do Programa de Pós-Graduação em Zootecnia - Universidade Estadual de Maringá, Bolsista do \\ CNPq. E-mail: marianaornaghi@hotmail.com \\ ${ }^{2}$ Departamento de Zootecnia e Ciência de Alimentos, Universidade de Zaragoza, Espanha. \\ ${ }^{3}$ Departamento de Zootecnia - Universidade Estadual de Londrina. \\ ${ }^{4}$ Professor Titular Zootecnia - Departamento de Zootecnia - Universidade Estadual de Maringá Pesquisador \\ Nivel $1 A-C N P q$.
}

\begin{abstract}
RESUMO. O consumo de carne bovina vem aumentado com o passar dos anos e com esse aumento cresce também a preocupação com a qualidade do produto, principalmente em relação á saúde. O que muito se fala na atualidade é da composição de ácidos graxos contido na carne bovina e os malefícios que podem causar o consumo desse alimento, o que pouco se fala é dos benefícios. Um dos fatores que influenciam diretamente na qualidade da carne bovina é a dieta consumida pelo animal. Com isso pesquisadores enfrentam grandes obstáculos procurando otimizar a qualidade do produto e assim como a eficiência em relação a produção animal. A glicerina vem sendo utilizada para alcançar esse objetivo na produção de bovinos de corte sendo possível sua adição na dieta substituindo o milho podendo reduzir o custo de produção. Este trabalho tem como objetivo realizar uma revisão bibliográfica da utilização da glicerina na dieta de bovinos e avaliar os resultados em relação à composição de ácidos graxos na carne.
\end{abstract}

Palavras chave: Biohidrogenação, Dieta, lipídeos, ruminantes, saúde.

\section{Glycerin as corn replacer in cattle nutrition and influence on the composition of fatty acids of meat: review}

\begin{abstract}
The beef consumption has increased over the years and consequently increases consumers' concern about quality of meat products, especially in relation to health. Currently composition of fatty acids and on beef and the not favorable consequences that can cause beef consumption were much commented, however benefits that have beef consumption have been little divulged. One of the factors that directly influence the quality of beef is the diet consumed by the animal. Researchers are trying to optimize product quality and optimize efficiency in relation to animal production. Glycerin has been used to achieve this goal in beef cattle production. Being possible add glycerin in the diet in order to replace corn and reducing cost of production. This paper aims to conduct a literature review of the use of glycerin in the diet of cattle and evaluate the results in relation to fatty acid composition in meat.
\end{abstract}

Key words: Biohydrogenation, diet, lipids, ruminant, health.

\section{Introdução}

O Brasil possui o maior rebanho comercial do mundo, com aproximadamente 208 milhões de cabeças de bovinos (ANUALPEC, 2014) e destaca-se como um dos maiores exportadores de carne bovina. Um dos principais sistemas de produção utilizado no âmbito nacional é o extensivo (pastejo) devido às condições climáticas favoráveis (Prado, 2010a, Ferraz \& Felício, 2010). Entretanto, nos períodos de baixa disponibilidade de alimentos ocorre a diminuição na produtividade dos animais, sendo necessário o estabelecimento de estratégias favoráveis ao ganho de peso dos animais e viáveis 
economicamente (Moreira \& Prado, 2010, Moreira et al., 2003, Moreira et al., 2004).

Os alimentos utilizados na alimentação de animais em confinamento é o fator mais limitante na utilização desta prática, pois compõe cerca de $80 \%$ do custo de produção (Silva et al., 2010). Entretanto, em períodos de críticos para a produção de forragem a intensificação do sistema produtivo permite tanto controlar a ingestão como fracionar o alimento a ser fornecido proporcionando melhor aproveitamento no rúmen aumentando absorção intestinal dos nutrientes e consequentemente prevenirem déficit dos mesmos (RC, 2000).

Um dos alimentos mais utilizado em confinamentos é o milho (Zea mays L.), que supre as exigências energéticas como também auxilia na manutenção protéica da ração (Prado, $\underline{2010 \mathrm{~b}})$. Apesar de o milho ser uma alternativa viável à nutrição animal a competição com a alimentação humana pode inviabilizar o custo e consequentemente a sua utilização na produção de ruminantes.

Segundo o ANUALPEC (2014), aproximadamente $\quad 68,5 \% \quad(38,7$ milhões de toneladas) da demanda interna nacional de milho grão foi destinada a alimentação animal. O maior consumo de milho dentre os setores da cadeia produtiva da carne destinaram-se a avicultura, suinocultura e bovinocultura, sendo produzidos aproximadamente 62 milhões de toneladas de ração (Sindirações, 2012). Segundo a Abimilho (2012) o baixo uso de substitutos ao milho na alimentação animal favorece o aumento no consumo de milho por parte da indústria de carnes, justificando o aumento observado no preço da saca de milho (87\%) dos últimos cinco anos (ANUALPEC, 2014).

Em busca por estratégias que permitam aumentar a prática de confinamentos, sem inviabilizar o custo de produção, tem-se aumentado a participação de co-produtos da agroindústria possíveis de utilização pelos ruminantes (Prado et al., 2010).

A glicerina, co-produto da produção do biodiesel, apresenta características com potencial para substituir o milho na alimentação dos ruminantes, sendo utilizada como fonte energética alternativa em dietas destinadas a terminação de bovinos (Farias et al., 2012, Françozo et al., 2013, Cruz et al., 2014, Silva et al., 2014). O glicerol presente na glicerina é utilizado pelos microrganismos ruminais para a formação de ácidos graxos voláteis de cadeia curta, ou ainda, pode ser absorvido e metabolizado na via glicolítica/gliconeogênica para manutenção dos níveis séricos de glicose (Kijora et al., 1998, Krehbiel, 2008).

No entanto, durante a fermentação de ácidos graxos oriundos de triglicerídeos ocorre a biohidrogenação dos ácidos graxos insaturados modificando o arranjo da sua estrutura molecular (Tamminga \& Doreau, 1991). Os ruminantes possuem baixa passagem dos ácidos graxos poliinsaturados (AGP) do rúmen para o intestino, pois ocorre a biohidrogenação pelas bactérias ruminais, principalmente, pelas Butyrivibrio fibrisolvens A38, Bifidobacterium breve e $B$. dentium. $\mathrm{O}$ processo de biohidrogenação caracteriza - se por um mecanismo de defesa dos microrganismos, pois os AGP são tóxicos às gram positivas e aos protozoários. Segundo Bonhomme (1990), o papel dos protozoários na biohidrogenação não é conclusivo e ainda esclarece que os protozoários ciliados são responsáveis por 30 a $40 \%$ da atividade lipolítica, além de participarem na hidrogenação de ácidos graxos. Tamminga \& Doreau (1991) afirmaram que há evidências da participação dos protozoários na biohidrogenação de ácidos graxos.

A biohidrogenação consiste na adição de um átomo de hidrogênio as duplas ligações da cadeia dos ácidos graxos, transformando os ácidos graxos insaturados em ácidos graxos saturados (Figura 1). Entretanto, Valinote et al. (2005) ressaltam que a biohidrogenação incompleta permite que o ácido linoléico conjugado (CLA) seja depositado na carne e leite dos ruminantes e assim, demonstrar suas propriedades anticarcinogênicas, de efeito antiinflamatório, sendo importante para a saúde do consumidor.

\section{Revisão bibliográfica}

Um dos principais fatores que influenciam na saúde do homem é a dieta consumida, sabendo que os produtos de origem animal são os principais responsáveis pelos ácidos graxos saturados em cerca de 60\% (Monteiro et al., 2000, Wood \& Enser, 1997, Wood et al., 1999, Wood et al., 2008).

Apesar do teor de gordura assustar em relação à saúde, não pode se esquecer de que o teor de lipídeos na carne é um fator importante influenciando na palatabilidade, suculência e 
textura da carne em caso da gordura intramuscular, já a subcutânea é necessária, pois atua como isolante térmico, protegendo a carcaça do encurtamento pelo frio, fenômeno que ocorre nas carcaças durante o resfriamento das mesmas nos frigoríficos (Wood et al., 2008, Hocquette et al., 2005).

Sendo assim uma das alternativas de se melhorar a composição de ácidos graxos (precursores do colesterol), pesquisadores buscam alimentos que quando adicionados na dieta de bovinos aumentem a composição de ácidos graxos insaturados, esses são benéficos a saúde do consumidor, pois aumentam os níveis de HDL (lipoproteína de alta densidade), responsáveis por desobstruí as artérias coronárias (Wood et al., 2003, Wood et al., 2004).

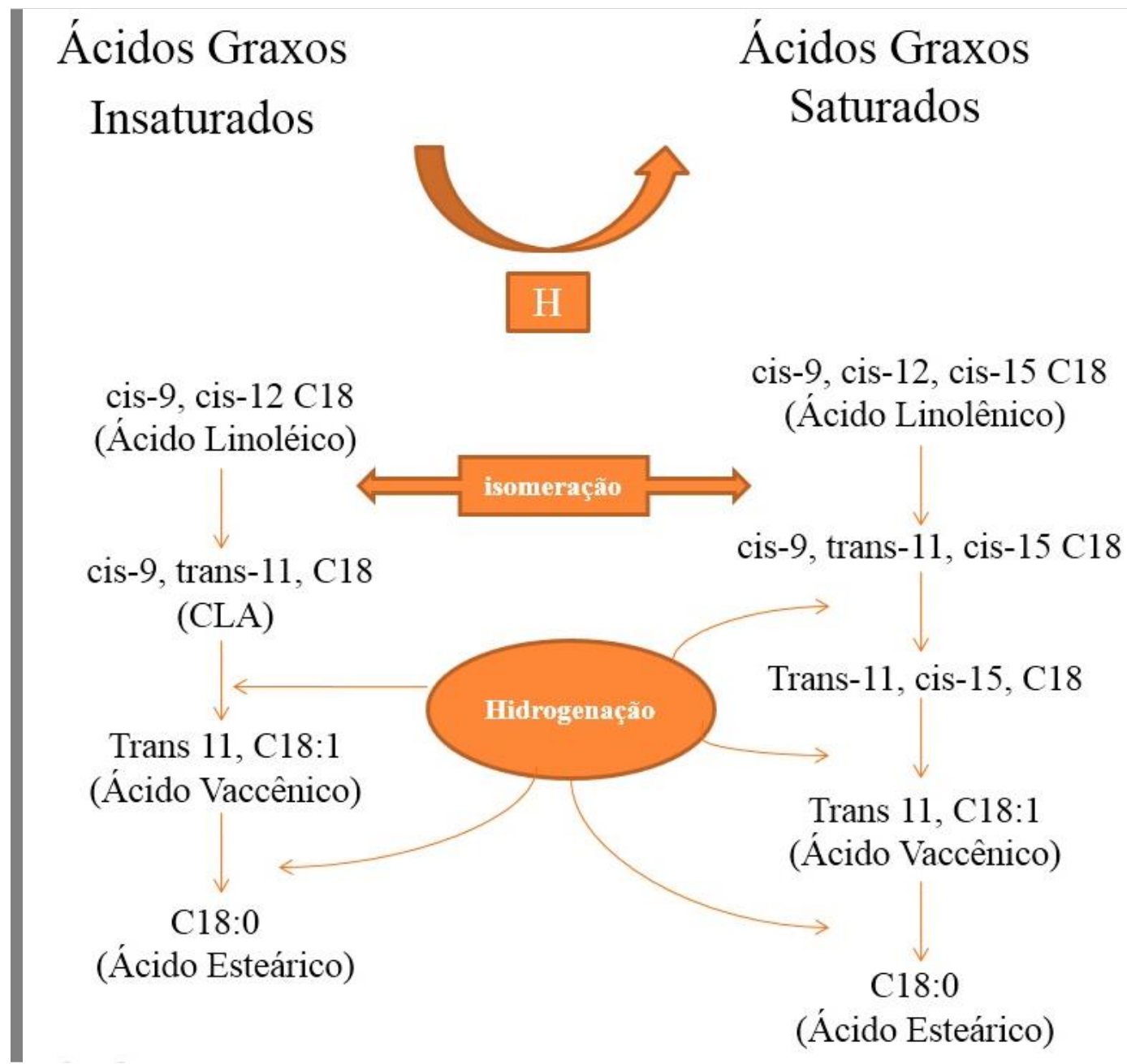

Figura 1. Mecanismo da biohidrogenação, transformação de ácido graxo insaturado em saturado.

Sabe - se que os principais fatores que influenciam a composição dos ácidos graxos são: grupo genético (Prado et al., 2008a, Prado et al., 2008b, Prado et al., 2008c), sistema de terminação (Aricetti et al., 2008, Padre et al., 2006), exercícios realizados, condições fisiológicas (Prado et al., 2009, Prado et al., 2014), sexo (Bretschneider, 2005), localização anatômica do músculo (Macedo et al., 2008) e nutrição (Rotta et al., 2009). No caso da nutrição uma das alternativas a ser testada é o uso da glicerina na nutrição animal que além de baratear o custo de produção que é elevado, pode ter capacidade de diminuir os teores de ácidos graxos saturados (AGS) e aumentar os ácidos graxos insaturados (AGI), característica muito importante para qualidade da carne.

O glicerol é um subproduto natural do processamento de óleos e gorduras, o que pode ser obtido mediante reação de saponifição de ácidos graxos. Apresenta uma grande quantidade de uso na indústria química e pode ser incluído na alimentação animal. É usado como umectante 
e para conservar alimentos como a ração animal. Participa na síntese de triacilgliceróis e fosfolipídeos além de atuar na via glicolítica e/ou gliconeogênese (Vokou et al., 1993).

Quando ácidos graxos insaturados com um número de 18 carbonos são ingeridos pelos animais ruminantes, grande quantidade é convertida em ácido graxo saturado C 18 (esteárico) pela ação das bactérias presentes no rúmen (Krehbiel, 2008).

Os ácidos graxos saturados quando consumidos em quantidades elevadas são fatores de risco à ocorrência de doenças cardiovasculares (Scollan et al., 2006), pois os níveis de lipoproteínas de baixa densidade (LDL) e lipoproteína de muito baixa densidade (VLDL) promovem o acumulo de gordura nas paredes arteriais causando doenças cardiovasculares.

\section{Glicerina na nutrição de ruminantes}

A produção de subprodutos que barateiam os custos de produção animal tem se tornado um grande desafio para pesquisadores. Um desses que visa diminuir os custos é a glicerina subproduto que pode substituir o milho como fonte energética na nutrição animal (Françozo et al., 2013, Eiras et al., 2014a, Eiras et al., 2014b). Além de alcançar economia na dieta de ruminantes e monogástricos reduz competição com a nutrição do homem e produção de combustível (principalmente nos EUA), que também é um fator que muito se fala nos dias atuais, preocupante pela produção de milho ser direcionada pela metade para produção de ração.

Glicerina um subproduto natural do processamento de óleos e gorduras, sendo $70 \%$ extraído do óleo de soja e os $30 \%$ restantes de sebo e gorduras animais e outras fontes (Oliveira et al., 2013), obtido da transesterificação, que consiste em separação da glicerina do óleo vegetal ou animal. A glicerina possui vários níveis de pureza dependendo do teor de metanol e de glicerol presentes. Dentre esses níveis obtém-se a glicerina de baixa pureza com $63.3 \%$ de glicerol, utilizada na adição do diesel comum; glicerina de média pureza $85 \%$, mais utilizada na alimentação animal e a pura com $99 \%$ de glicerol, utilizada na indústria farmacêutica e alimentação humana (Ferraro et al., 2009).

A glicerina de média pureza apresenta uma grande quantidade de uso na indústria química $\mathrm{e}$ pode ser incluída na alimentação animal, na tabela 1 podemos observar a composição química da glicerina de média pureza. Pode ser usada como umectante e para conservar alimentos como a ração animal. Participa na síntese de triacilgliceróis e fosfolipídeos além de atuar na via glicolítica para manutenção dos níveis séricos de glicose (Gomes et al., 2011).

Co-produto da produção do biodiesel apresenta características com potencial para substituir o milho na alimentação dos ruminantes. A glicerina pode ser utilizada como uma fonte energética alternativa na alimentação animal, particularmente para ruminantes (Eiras et al., $\underline{2013}$, Farias et al., 2012, Françozo et al., 2013)

Tabela 1 Composição química da glicerina

\begin{tabular}{lr}
\hline Parâmetros & Resultados \\
\hline Água* & $23,2 \mathrm{~g} / \mathrm{kg}$ \\
Cinzas & $47,6 \mathrm{~g} / \mathrm{kg}$ \\
Glicerol & $812 \mathrm{~g} / \mathrm{kg}$ \\
Metanol & $3,32 \mathrm{mg} / \mathrm{kg}$ \\
Sódio & $11,6 \mathrm{~g} / \mathrm{kg}$ \\
Potássio & $79,1 \mathrm{mg} / \mathrm{kg}$ \\
Cloreto & $35,8 \mathrm{mg} / \mathrm{kg}$ \\
Magnésio & $16,3 \mathrm{mg} / \mathrm{kg}$ \\
Fósforo & $239 \mathrm{mg} / \mathrm{kg}$ \\
Energia Bruta & $14,2 \mathrm{KJ}$ \\
\hline
\end{tabular}

Realizado pelo Instituto de Tecnologia do Paraná TECPAR, Divisão Biodiesel, em Curitiba, Paraná.*Karl Fischer.

O glicerol é fermentado principalmente a ácido propiônico precursor da deposição muscular, muito interessante na produção de bovinos, pois reduz conseqüentemente a produção de metano uma vez que animais alimentados com dietas que proporcionam maior proporção de propionato no rúmen tendem a diminuir a produção de metano, pois produção de propionato envolve o consumo de $\mathrm{H}_{2}$ e não o contrário como ocorre na produção de AG acético e butírico, assim economizando energia para produção final como carne e leite (Krehbiel, 2008).

O fenômeno mais importante que acontece com os ácidos graxos derivados dos triglicerídeos é a biohidrogenação dos ácidos graxos insaturados. Quando ácidos graxos insaturados C 18 (oléico, linoléico e linolênico contendo uma, duas e três duplas ligações, respectivamente) são colocados no rúmen, grande quantidade é convertida em ácido graxo saturado C18 (esteárico), sendo fermentado no rúmen em ácidos graxos voláteis e metabolizado no fígado convertido em glicose e aproveitado pelo animal como fonte energética (Kijora et al., 1998). 
Gomes et al. (2011) observaram ser possível a adição de até $30 \%$ de glicerol na dieta total (100\% de substituição do milho) de cordeiros em confinamento durante 60 dias sem alterar o desempenho animal, ingestão de alimentos e rendimento dos cortes nobres de cordeiros. Farias et al. (2012) com adição de níveis de glicerina $(0,0 ; 3,8 ; 6,1$ e $9,0 \%)$ observaram redução no ganho médio diário a medida que aumentara os níveis de inclusão de glicerina na dieta.

Françozo et al. (2013) não observaram alterações no desempenho animal e características de carcaça de novilhos Nelores alimentados com diferentes níveis de glicerina $(0$, 5 e $12 \%$ ) terminados em confinamento, indicando a viabilidade de utilização do co-produto na terminação de bovinos. Apesar dessa afirmação deve ser feita uma análise do preço e porcentagem a ser adicionada de fonte protéica, pois quando a dieta tem substituição do milho pela glicerina devem ser adicionados níveis maiores do percentual protéico sabendo que a glicerina tem diminuto teor de proteína $(0.07 \%$ em média), considerado não significativo.

\section{Ácidos graxos}

Quando se fala em ingestão de alimentos pelo homem, as consequências relacionadas á saúde é uma questão de grande importância observada nos dias atuais pelos consumidores do mundo todo. A carne é um produto que muito se consome e é polemica ao se falar em saúde, pois sua composição de ácidos graxos é relacionada aos níveis séricos de colesterol. Sendo um fator preocupante os níveis de colesterol no sangue são observados cada vez mais ao passar dos dias. O que se entende sobre o colesterol é produto de pouca informação, pois o colesterol não tem somente a função de causar doenças como a aterosclerose e a hipertensão. Segundo $\underline{\text { Scollan et }}$ al. (2006) o colesterol é um importante componente na dieta dos seres humanos, auxiliando na produção hormonal das glândulas sexuais e adrenais e no transporte e absorção das vitaminas lipossolúveis.

O colesterol está diretamente relacionado com a dieta e também é dependente da quantidade desses alimentos a serem ingeridos, ou seja, os níveis e tipo do colesterol no sangue irão depender do tipo de alimento e quantidade consumida, podendo ser ou não prejudicial à saúde. O grande desafio de pesquisadores é reduzir os riscos do colesterol com propriedades prejudiciais ao homem, visando melhorar a qualidade de vida e longevidade dos seres humanos.

Os ácidos graxos na maioria das vezes apresentam cadeias de 14,16 ou 18 carbonos podendo apresentar ou não duplas ligações, aqueles que não apresentam dupla ligação são chamados de ácidos graxos saturados os que têm duas ligações são os insaturados. Dentro do grupo de insaturados temos os monoinsaturados com uma dupla ligação e os poliinsaturados com duas ou mais ligações. Dentre os poliinsaturados estão alguns dos essenciais necessários para o bom funcionamento do organismo, o ácido linoléico seria um bom exemplo de essencial que a partir dele permite ser convertido em ácido gama linoléico e ácido araquidônico (Martin et al., 2006).

Sendo a carne bovina uma das mais consumidas mundialmente, uma ferramenta chave para melhorar a qualidade deste alimento seria aumentar o teor de ácidos graxos poliinsaturados (AGPI), pois esses são benéficos para saúde sendo precursores das HDL (lipoproteínas de alta densidade) que desobstruem as artérias coronárias reduzindo riscos de aterosclerose, ou seja, os ácidos graxos poli-insaturados oferecem proteção ao sistema cardiovascular (Morais et al., 2010).

Os ácidos graxos saturados (AGS) quando consumidos em quantidades elevadas são fatores de risco à ocorrência de doenças cardiovasculares, pois os níveis de lipoproteínas de baixa densidade (LDL) e lipoproteína de muito baixa densidade (VLDL) promovem o acumulo de gordura nas paredes arteriais causando doenças cardiovasculares (Funck et al., 2006). Além de influenciar na saúde do ser humano os teores de ácidos graxos influenciam nas características físicas, químicas e organolépticas da carne.

A gordura da carne bovina apresenta cerca de $48 \%$ de gordura saturada e $52 \%$ de gordura insaturada (Rotta et al., 2009). Sendo os AGS menos desejáveis o ácido palmítico (C16:0) e ácido miristico (C14:0) segundo JiménezColmenero et al. (2001) indicam o ácido mirístico como mais prejudicial a saúde pelos efeitos hipercolesterolêmicos. Uma vez que os ácidos graxos saturados são associados à problemas de saúde, algumas pesquisas manipulam a dieta animal visando melhorar a composição de ácidos graxos na carne bovina e 
consequentemente a qualidade de vida de quem a consome.

Os ruminantes são animais interessantes pois a composição de ácidos graxos da dieta não é a mesma que encontramos no produto final, pois os microrganismos presentes na flora ruminal são capazes de fazer a biohidrogenação dos ácidos graxos insaturados (AGI), basicamente adicionam uma molécula de hidrogênio a cadeia dos AGI transformando os em saturados.

A biohidrogenação pelas bactérias ruminais; pode ser considerada um mecanismo de defesa desses microrganismos, pois os AGPI são tóxicos para eles, principalmente para as bactérias gram positivas e protozoários. $\mathrm{O}$ papel dos protozoários na biohidrogenação não é conclusivo. Um dos produtos da biohidrogenação ruminal é o ácido linoleico conjugado (CLA) que possui propriedades anticarcinogênicas e efeito antiinflamatório que evitam doenças renais, sendo importante para a saúde do consumidor.

\section{Ácido Linoléico Conjugado (CLA)}

É produto da biohidrogenação incompleta do ácido linoléico pelas bactérias do rúmen principalmente pela Butyrivibrio fibrisolvens, normalmente, a biohidrogenação acontece de forma completa; porém, alguns produtos intermediários podem atravessar a parede do rúmen e serem utilizados na síntese de lipídios no tecido mamário e adiposo (Bauman et al., 1999).

A extensão da biohidrogenação depende da natureza das dietas: com concentrado maior que $70 \%$, ela cai para $50 \%$ e $65 \%$, respectivamente para linoléico e linolênico, a queda no $\mathrm{pH}$ normalmente associada com este tipo de dieta reduz a lipólise, passo essencial para que ocorra a biohidrogenação (Tamminga \& Doreau, 1991). Beam et al. (2000) não observaram efeito na taxa de biohidrogenação na dieta oferecida a uma vaca ou o tempo após o fornecimento do alimento, mas sim para a fonte de lipídeos e a quantidade destes.

O ácido linoléico conjugado chamado CLA (C18:2 c-9 t-11) é produzido em baixas concentrações $(<0.01 \%)$ e em sua maioria é encontrado nos produtos de origem bovina como carne e leite. O cis 9, trans 11 é o isômero predominante na carne de ruminantes mas em menor quantidade quando comparado ao leite (Bauman et al., 1999) possui propriedades de alto valor em relação a saúde, um anticarcinogênico comprovado (Beam et al., 2000). Subsequente trabalho encontrou que dietas com CLA podem reduzir a incidência de tumores malignos em animais, modelos para a glândula mamária, estômago, cólon, e tumores de pele (MoyaCamarena \& Belury, 1999).

\section{Conclusão}

Glicerina pode substituir o milho na nutrição animal sendo uma fonte energética eficiente, pois economiza na produção de gás metano, consequentemente proporcionando maior produção de energia liquida utilizada na produção do animal. Em níveis propostos pode diminuir o teor de ácidos graxos saturados que são maléficos a saúde do consumidor final, e aumentar o teor de ácidos graxos insaturados que ajuda a eliminar o colesterol diminuindo a incidência de aterosclerose.

\section{Referências Bibliográficas}

ANUALPEC. 2014. Anuário da Pecuária Brasileira, 20th edn. Instituto FNP, São Paulo, SP, Brasil.

Aricetti, J. A., Rotta, P. P., Prado, R. M., Perotto, D., Moletta, J. L., Matsushita, M. \& Prado, I. N. 2008. Carcass characteristics, chemical composition and fatty acid profile of Longissimus muscle of bulls and steers finished in a pasture system. AsianAustralasian Journal of Animal Sciences, 21, 1441-1448.

Bauman, D. E., L.H., B., Corl, B. A. \& Griinari, J. M. 1999. Biosynthesis of conjugated linoleic acid in ruminants. Proceedings of the American Society of Animal Science, 1, 1-15.

Beam, T. M., Jenkins, T. C., Moate, P. J., Kohn, R. A. \& Palmquist, D. L. 2000. Effects of amount and source of fat on the rates of lipolysis and biohydrogenation of fatty acids in ruminal contents. Journal of Dairy Science, 83, 2564-2573.

Bonhomme, A. 1990. Rumen ciliates: their metabolism and relationships with bacteria and their hosts. Animal Feed Science and Technology, 30, 203-266.

Bretschneider, G. 2005. Effects of age and method of castration on performance and stress response of beef male cattle: A review. Livestock Production Science, 97, 89-100.

Cruz, O. T. B., Valero, M. V., Zawadzki, F., Rivaroli, D. C., Prado, R. M., Lima, B. S. \& 
Prado, I. N. 2014. Effect of glycerine and essential oils (Anacardium occidentale and Ricinus communis) on animal performance, feed efficiency and carcass characteristics of crossbred bulls finished in a feedlot system. Italian Journal of Animal Science, 13, 790797.

Eiras, C. E., Barbosa, L. P., Marques, J. A., Lima, B. S., Zawadzki, F., Perotto, D. \& Prado, I. N. 2014a. Glycerine levels in the diets of crossbred bulls finished in feedlot: apparent digestibility, feed intake and animal performance. Animal Feed Science and Technology, 197, 222-226.

Eiras, C. E., Marques, J. A., Prado, R. M., Valero, M. V., Bonafé, E. G., Zawadzki, F., Perotto, D. \& Prado, I. N. 2014b. Glycerin levels in the diets of crossbred bulls finished in feedlot: Carcass characteristics and meat quality. Meat Science, 96, 930-936.

Eiras, C. E., Marques, J. A., Torrecilhas, J. A., Zawadzki, F., Moletta, J. L. \& Prado, I. N. 2013. Glycerin levels in the diets of crossbred bulls finished in feedlot: ingestion behavior, feeding intake and ruminal efficiency. Acta Scienciarum. Animal Sciences, 35, 411-416.

Farias, M. S., Prado, I. N., Valero, M. V., Zawadzki, F., Silva, R. R., Eiras, C. E., Rivaroli, D. C. \& Lima, B. S. 2012. Níveis de glicerina para novilhas suplementadas em pastagens: desempenho, ingestão, eficiência alimentar e digestibilidade. Semina: Ciências Agrárias, 33, 1177-1188.

Ferraro, S. M., Mendoza, G. D., Miranda, L. A. \& Gutiérrez, C. G. 2009. In vitro gas production and ruminal fermentation of glycerol, propylene glycol and molasses. Animal Feed Science and Technology, 154, 112-118.

Ferraz, J. B. S. \& Felício, P. E. 2010. Production systems - An example from Brazil. Meat Science, 84, 238-243.

Françozo, M. C., Prado, I. N., Cecato, U., Valero, M. V., Zawadzki, F., Ribeiro, O. L., Prado, R. M. \& Visentainer, J. V. 2013. Growth performance, carcass characteristics and meat quality of finishing bulls fed crude glycerinesupplemented diets. Brazilian Archives of Biology and Technology, 56, 327-336.

Funck, L. G., Barrera-Arellano, D. \& Block, J. M. 2006. Ácido linoléico conjugado (CLA) e sua relação com a doença cardiovascular e os fatores de risco associados. Archivos Latinoamericanos de Nutrición, 56, 123-134.

Gomes, M. A. B., Moraes, G. V., Mataveli, M., Macedo, F. A. F., Carneiro, T. C. \& Rossi, R. M. 2011. Performance and carcass characteristics of lambs fed on diets supplemented with glycerin from biodiesel production. Revista Brasileira de Zootecnia, 40, 2211-2219.

Hocquette, J. F., Richardson, R. I., Prache, S., Medale, F., Duffy, G. \& Scollan, N. D. 2005. The future trends for research on quality and safety of animal products. Italian Journal of Animal Science, 4, 49-72.

Jiménez-Colmenero, F., Carballo, J. \& Cofrades, S. 2001. Healthier meat and meat products: their role as functional foods. Meat Science, 59, 5-13.

Kijora, C., Bergner, H., Götz, K. P., Bartelt, J., Szakacs, J. \& Sommer, A. 1998. Reserach note: Investigation on the metabolism of glycerol in the rumen of bulls. Arch Tieremahr, 51, 341-348.

Krehbiel, C. R. 2008. Ruminal and physiological metabolism of glycerin. Journal of Animal Science, E86 (Supplement), 392 (Abstr.).

Macedo, L. M., Prado, I. M., Prado, J. M., Rotta, P. P., Prado, R. M., Souza, N. E. \& Prado, I. N. 2008. Chemical composition and fatty acids profile of five carcass cuts of crossbred heifers finished in feedlot. Semina: Ciências Agrárias, 29, 597-608.

Martin, C. A., Almeida, V. V. d., Ruiz, M. R., Visentainer, J. E. L., Matshushita, M., Souza, N. E. d. \& Visentainer, J. V. 2006. Omega-3 and omega- 6 polyunsaturated fatty acids: importance and occurrence in foods. Revista de Nutrição, 19, 761-770.

Monteiro, C. A., Mondini, L. \& Costa, R. B. 2000. Mudanças na composição e adequação nutricional da dieta familiar nas áreas metropolitanas do Brasil (1988-1996). Revista de Saúde Pública, 34, 251-8.

Morais, D. R., Visentainer, J. E. L., Santos, L. P., Matsushita, M., Souza, N. E. \& Visentainer, J. V. 2010. Evaluation of lipid extraction and fatty acid composition of human plasma. Avaliação da extração lipídica e composição em ácidos graxos do plasma humano, 32, 439-443. 
Moreira, F. B. \& Prado, I. N. 2010. Sazonalidade na produção e qualidade de plantas forrageiras. In: Prado, I. N. (ed.) Produção de bovinos de corte e qualidade da carne. Eduem, Maringá, Paraná, Brasil.

Moreira, F. B., Prado, I. N., Cecato, U., Wada, F. Y., Nascimento, W. G. \& Souza, N. E. 2003. Suplementação com sal mineral proteinado para bovinos de corte, em crescimento e terminação, mantidos em pastagem de grama estrela roxa (Cynodon plectostachyrus Pilger) no inverno. Revista Brasileira de Zootecnia, 32, 449-455.

Moreira, F. B., Prado, I. N., Cecato, U., Zeoula, L. M., Wada, F. Y. \& Torii, M. S. 2004. Níveis de suplementação com sal mineral proteinado para novilhos Nelore terminados em pastagem no período de baixa produção forrageira. Revista Brasileira de Zootecnia, 33, 1814-1821.

Moya-Camarena, S. Y. \& Belury, M. A. 1999. Species differences in the metabolism and regulation of gene expression by conjugated linoleic acid. Nutrition reviews, 57, 336-340.

NRC. 2000. Nutrient Requirements of Beef Cattle, 7th rev. edn. Natl. Acad. Press, Washington, DC, USA.

Oliveira, J. S., Antoniassi, R., Freitas, S. C. \& Müller, M. D. 2013. Chemical composition of glycerin produced by biodiesel plants in Brazil and potential utilization in animal feeding. Ciência Rural, 43, 509-512.

Padre, R. G., Aricetti, J. A., Moreira, F. B., Mizubuti, I. Y., Prado, I. N., Visentainer, J. V., Souza, N. E. \& Matsushita, M. 2006. Fatty acid profile, and chemical composition of Longissimus muscle of bovine steers and bulls finished in pasture system. Meat Science, 74, 242-248.

Prado, I. N. 2010a. Introdução a bovinocultura de corte. In: Prado, I. N. (ed.) Produção de bovinos de corte e qualidade da carne. Eduem, Maringá, Paraná, Brasil.

Prado, I. N. 2010b. Produção de bovinos de corte $e$ qualidade da carne. Eduem, Maringá, Paraná, Brasil.

Prado, I. N., Aricetti, J. A., Rotta, P. P., Prado, R. M., Perotto, D., Visentainer, J. V. \& Matsushita, M. 2008a. Carcass characteristics, chemical composition and fatty acid profile of the Longissimus muscle of bulls (Bos taurus indicus vs. Bos taurus taurus) finished in pasture systems. Asian-Australasian Journal of Animal Sciences, 21, 1449-1457.

Prado, I. N., Campo, M. M., Muela, E., Valero, M. V., Catalan, O., Olleta, J. L. \& Sañudo, C. 2014. Effect of castration age, protein level and lysine/methionine ratio in the feed on animal performance, carcass and meat quality of Friesian steers intensively reared. Animal, $8,1-8$.

Prado, I. N., Marques, J. A., Rotta, P. P., Prado, R. M., Visentainer, J. V. \& Souza, N. E. 2009. Meat quality of the Longissimus muscle of bulls and steers ( $1 / 2$ Nellore vs. $1 / 2$ Simmental) finished in feedlot. Journal of Animal and Feed Sciences, 18, 221-230.

Prado, I. N., Moreira, F. B., Prado, R. M. \& Rotta, P. P. 2010. Alimentos usados para supelementação de bovinos de corte. In: Prado, I. N. (ed.) Produção de bovinos de corte e qualidade da carne. Eduem, Maringá, Paraná, Brasil.

Prado, I. N., Prado, R. M., Rotta, P. P., Visentainer, J. V., Moletta, J. L. \& Perotto, D. 2008b. Carcass characteristics and chemical composition of the Longissimus muscle of crossbred bulls (Bos taurus indicus vs Bos taurus taurus) finished in feedlot. Journal of Animal and Feed Sciences, 17, 295-306.

Prado, I. N., Rotta, P. P., Prado, R. M., Visantainer, J. V., Moletta, J. L. \& Perotto, D. 2008c. Carcass characteristics and chemical composition of the Longissimus muscle of Purunã and 1/2 Purunã vs. 1/2 Canchin bulls. Asian-Australasian Journal of Animal Sciences, 21, 1296-1302.

Rotta, P. P., Prado, R. M., Prado, I. N., Valero, M. V., Visentainer, J. V. \& Silva, R. R. 2009. The effects of genetic groups, nutrition, finishing systems and gender of Brazilian cattle on carcass characteristics and beef composition and appearance: a review. AsianAustralasian Journal of Animal Sciences, 22, 1718-1734.

Scollan, N., Hocquette, J. F., Nuernberg, K., Dannenberger, D., Richardson, I. \& Moloney, A. 2006. Innovations in beef production systems that enhance the nutritional and health value of beef lipids and their relationship with meat quality. Meat Science, 74, 17-33. 
Silva, L. G., Torrecilhas, J. A., Ornaghi, M. G., Eiras, C. E., Prado, R. M. \& Prado, I. N. 2014. Glycerin and essential oils in the diet of Nellore bulls finished in feedlot: animal performance and apparent digestibility. Acta Scienciarum. Animal Sciences, 36, 177-184.

Silva, R. R., Prado, I. N., Carvalho, G. G. P., Silva, F. F., Almeida, V. V. S., Santana Júnior, H. A., Paixão, M. L. \& Abreu Filho, G. 2010. Níveis de suplementação na terminação de novilhos Nelore em pastagens: aspectos econômicos. Revista Brasileira de Zootecnia, 39, 2091-2097.

Tamminga, S. \& Doreau, M. 1991. Lipids and rumen digestion. In: Jouany, J. P. (ed.) Rumen microbial metabolism and ruminant digestion. Institut National de la Recherche Agronomique, Paris, FR.

Valinote, A. C., Nogueira Filho, J. C. M., Leme, P. R., Silva, S. D. E. \& Cunha, J. A. 2005. Fontes de lipídeos e monensina na alimentação de novilhos Nelore e sua relação com a população de protozoários ciliados do rúmen. Revista Brasileira de Zootecnia, 34, 1418-1423.

Vokou, D., Kokkini, S. \& Bessiere, J. M. 1993. Geographic variation of Greek oregano (Origanum vulgare ssp. hirtum) essential oils. Biochemical Systematics and Ecology, 21, 287-295.
Wood, J. D. \& Enser, M. 1997. Factors influencing fatty acids in meat and the role of antioxidants in improving meat quality. British Journal of Nutrition, 78, S49-S60.

Wood, J. D., Enser, M., Fisher, A. V., Nute, G. R., Richardson, R. I. \& Sheard, P. R. 1999. Manipulating meat quality and composition. Proceedings of the Nutrition Society, 58, 363370.

Wood, J. D., Enser, M., Fisher, A. V., Nute, G. R., Sheard, P. R., Richardson, R. I., Hughes, S. I. \& Whittington, F. M. 2008. Fat deposition, fatty acid composition and meat quality: A review. Meat Science, 78, 343-358.

Wood, J. D., Enser, M., Fisher, A. V., Nute, G. R., Whittington, F. M. \& Richardson, R. I. 2003. Effects of diets on fatty acids and meat quality. Options Méditerranéennes, Series A, 67, 133-141.

Wood, J. D., Richardson, R. I., Nute, G. R., Fisher, A. V., Campo, M. M., Kasapidou, E., Sheard, P. R. \& Enser, M. 2004. Effects of fatty acids on meat quality: a review. Meat Science, 66, 21-32.

Recebido em Janeiro 6, 2016

Aceito em Fevereiro 5, 2016

License information: This is an open-access article distributed under the terms of the Creative Commons Attribution License, which permits unrestricted use, distribution, and reproduction in any medium, provided the original work is properly cited. 